Journal of

Cancer Research and Therapeutic Oncology

\title{
Current Status and Future Perspectives of Anti-Angiogenic Therapeutic Attempts for Glioblastoma
}

\author{
Maria Patrizia Mongiardi ${ }^{1}$, Roberto Pallini ${ }^{2}$, Andrea Levi ${ }^{1}$, Maria Laura Falchetti ${ }^{1{ }^{*}}$ \\ ${ }^{1}$ CNR-IBBC, Institute of Biochemistry and Cell Biology, Via Ercole Ramarini 32-00015 Monterotondo Scalo, Rome, Italy \\ ${ }^{2}$ Institute of Neurosurgery, Fondazione Policlinico Universitario Agostino Gemelli IRCCS, Università Cattolica del Sacro \\ Cuore, Largo Agostino Gemelli 8-00168, Rome, Italy
}

${ }^{*}$ Corresponding author: Maria Laura Falchetti, CNR-IBBC, Institute of Biochemistry and Cell Biology, Italy, Tel:+39 06 90091469, email: marialaura.falchetti@cnr.it

Received Date: January 09, 2019 Accepted Date: February 13, 2020 Published Date: February 17, 2020

Citation: Maria Patrizia Mongiardi (2020) Current Status and Future Perspectives of Anti-Angiogenic Therapeutic Attempts for Glioblastoma. J Cancer Res Therap Oncol 8: 1-9.

\begin{abstract}
Glioblastoma, the most aggressive brain tumor, is associated with invariably poor prognosis in spite of extensive surgical resection, radiotherapy, and concomitant and adjuvant chemotherapy. The histological landmarks of glioblastoma are massive necrosis and prominent angiogenesis. Glioblastoma vasculature is structurally and functionally aberrant, characterized by tortuous and leaky vessels, with an increased diameter and significantly thickened basement membranes. This altered vasculature enhances tumor hypoxia and affects the possibility of effective drug delivery to the tumor. Many efforts have been spent in developing therapeutic strategies targeting glioblastoma neo-angiogenesis, with the dual aim of inhibiting tumor growth and stabilizing tumor vasculature, therefore improving chemotherapy delivery to the tumor. Bevacizumab, a humanized monoclonal antibody targeting Vascular Endothelial Growth Factor (VEGF), has been approved by Food and Drug Administration (FDA) for recurrent glioblastoma, but unfortunately it seems to have limited efficacy in terms of overall survival. Here, we review literature data both from molecular and clinical studies and analyze the state of the art and the future perspectives of antiangiogenic therapies for glioblastoma.
\end{abstract}

Keywords: glioblastoma; antiangiogenic therapies

(C)2020 The Authors. Published by the JScholar under the terms of the Creative Commons Attribution License http://creativecommons.org/licenses/ by/3.0/, which permits unrestricted use, provided the original author and source are credited. 


\section{Introduction}

Glioblastoma is the most malignant primary brain tumor in adults with an estimated 12,000 cases annually in the United States. In most European countries, new cases of glioblastoma occur in approximately $2-3$ people in every 100,000 each year. Despite improvements in the median and short-term overall survival shown in recent large clinical trials for glioblastoma, the percentage of patients with glioblastoma achieving 5 -year overall survival remains very low $[1,2]$. According to the most recent World Health Organization classification [3], glioblastomas can be classified into two main classes referred to as isocitrate dehydrogenase gene (IDH) status. Glioblastoma, IDHwild type (about $90 \%$ of cases), is the most common glioblasto$\mathrm{ma}$ in patients over 55 years of age, also referred to as de novo or primary glioblastoma. Glioblastoma, IDH-mutant (about $10 \%$ of cases), is typically a tumor developing from lower-grade gliomas, more frequent in the younger population [4]. A third class comprises a low percentage of glioblastoma where a full IDH characterization is not possible.

The current standard of care for glioblastoma patients envisages surgery followed by adjuvant radiation and chemotherapy with the alkylating cytotoxic agent temozolomide. Overthe past 20 years, despite the innovation of surgery, radiotherapy, and chemotherapy, glioblastoma dismal prognosis has not significantly improved. The tumor invariably recurs after surgery, leading patients to die within 15-17 months [1]. Tumor recurrence after surgery depends, at least in part, on the tumor's highly infiltrative nature and on the consequent impossibility to perform a complete surgical resection of the tumor mass. Residual presence of microscopic foci leads to relapse or even recurrence of the disease. The presence of wide necrotic areas and extensive neo-angiogenesis are the histological landmarks of glioblastoma [5]. Neo-angiogenesis is a peculiar histological feature of glioblastoma, which is a tumor paradigmatic in its ability to induce neo-angiogenesis. Solid tumors cannot grow beyond a few millimeters diameter in the absence of adequate oxygen and nutrient supply. In the early seventies of the last century, Dr. Folkman, a pioneer in the studies on neoplastic angiogenesis, observed that solid tumors exhibit diffuse vascular network, tortuous, leaky and hemorrhagic, and that poorly vascularized tumor are unable to grow beyond $2 \mathrm{~mm}$, a size at which diffusion of oxygen and nutrients becomes limiting [6]. Fifty years later, it is well established that a crucial event in the early phases of solid tumors growth is the so-called angiogenic switch. This complex phenomenon implies a change in the balance between pro- and anti-angio- genic factors, with the resultant boost towards a pro-angiogenic outcome (Figure1). The angiogenic switch allows the tumor mass to grow beyond a critical size, exit a dormancy state of a vascularized hyperplasia and eventually results in malignant tumor progression [7].

Tumor angiogenesis is a multistep process, which starts when a global up-regulation of pro-angiogenic factors triggers endothelial cell proliferation, followed by degradation of extracellular matrix, proliferation, and migration toward tumor microenvironment [8]. Several factors act as regulators of angiogenesis, such as Vascular Endothelial Growth Factor (VEGF), Fibroblast Growth Factor (FGF), Platelet-Derived Growth Factor (PDGF), integrins, angiopoietins, and interleukin-8 $[9,10$, 11] (Figure 1). Among these factors, VEGF and its receptors play a fundamental role [12]. Mammalian genome, including humans, encodes for five VEGF family members (VEGF-A, -B, -C, -D, Placental Growth Factor).VEGF-A and its receptorsVEGFR-1(encoded by FLT 1 gene) and VEGF-2 (encoded by KDR gene) play major roles both in physiological and pathological angiogenesis, including tumor angiogenesis. VEGF-A binds to and activates VEGFR-1 and VEGFR-2, promoting angiogenesis, vascular permeability, cell migration, and modulating gene expression [13]. Further, an autocrine loop of VEGF-A and its receptors contributes to vascular endothelial cell functions [14]. VEGFR-1 affinity for VEGF-A is about 10 fold higher than that of VEGFR-2, whereas its tyrosine kinase activity is approximately 10 -fold lower than that of VEGFR-2 [12, 15]. The major proangiogenic signal is generated from the ligand-activated VEGFR-2. The VEGFA mRNA undergoes alternative splicing, originating mature transcripts which are translated in several peptides differing in length. In humans, we find peptides of 121, 165, 189, and 206 amino acids [16]. VEGFA165 is the most important VEGFA subtype, both for quantity and function importance. It binds to VEGF receptors 1 and 2, activating their tyrosine kinase activity and promoting angiogenesis, vascular permeability, cell migration and gene expression [13].

Glioblastoma vasculature is structurally and functionally aberrant, characterized by tortuous and leaky vessels, with an increased diameter and significantly thickened basement membranes. This altered vasculature enhances tumor hypoxia and affects the possibility of effective drug delivery to the tumor [17]. Of note, blood-brain barrier (BBB) integrity is preserved in the majority of vessels located outside the tumor bulk, and the tumor cells that invade the brain travel along vessels with retained BBB integrity [18]. 
Normal Angiogenesis

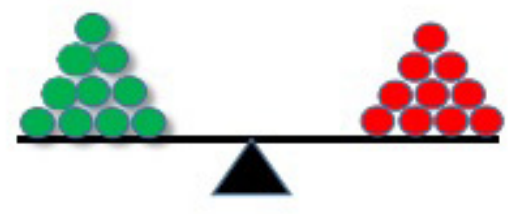

\section{Neoplastic Angiogenesis}

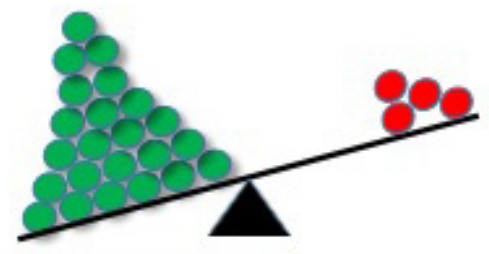

Angiogenic switch

Pro-Angiogenic Factors

VEGF (Vascular Endothelial Growth Factor) bFGF (basic Fibroblast Growth Factor)

PIGF (Placental Growth Factor)

G-CSF (Granulocyte-Colony-Stimulating Factor)

HGF (Hepatocyte Growth Factor)

PDGF (Platelet-Derived Growth Factor)

Angiopoletin 1,2

TGF $\beta$ (Transforming Growth Factor $\beta$ )

IL1, 3, 8 (Interleukin 1, 3, 8)

COX2 (Cyclooxygenase 2)

Tie2 (Tyrosine Kinase with immunoglobulin like and EGF like domains 2)

MMP (Matrix Metalloproteinases)

NOS (Nitric Oxide Sinthase)

GCSF (Granulocyte-Colony Stimulating Factor)

IF $\mathrm{N}$ - $\boldsymbol{\alpha}$ (Interferon- $\alpha)$

IGF (Insulin-like Growth Factor)
Anti-Angiogenic Factors

PF4 (Platelet Factor 4)

BAl1 (Brain-specific Angiogenesis Inhibitor 1)

Endostatin

Vasostatin

Troponin 1

Throbospondin

TIMP (Metallopeptidase Inhibitor)

PAI (Plasminogen Activator Inhibitor-1)

Angiostatin

TSP-1 (Thrombospondin type 1)

IL-4, 10, 12 (Interleukin-4, 10, 12)

PEX (Hemopexin-like domain)

Arresten

Canstatin

PEDF (Pigment Epithelium-Derived Factor)

Figure 1. Tumor-associated angiogenic switch. A tumor-triggered imbalance between pro- and anti-angiogenic factors results in an angiogenic switch that leads to tumor neovascularization.

Angiogenesis can be triggered by hypoxia-dependent as well as independent mechanisms. Hypoxia stabilizes Hypoxia Inducible Factor-1 $\alpha$ (HIF-1 $\alpha$ ) protein, a crucial transcription factor for cellular adaptation to low oxygen tension, which up regulates VEGF gene transcription [19]. Conversely, altered activation of mitogenic and survival pathways, as Ras/mitogen-activated protein kinase, can influence VEGF expression independently from hypoxia and HIF-1a. Many experimental evidences address a role for VEGF in promoting the proliferation of glioblastoma stem-like cells [20]. In addition, stem-like glioma cells significantly secrete VEGF and other pro-angiogenic factors $[21,22]$. As an obvious consequence, an antiangiogenic therapy might result in doubly effective since it could target neo-angiogenic phenomena and limit the proliferation of tumor cells at once. In addition, as at first proposed by Jain in 2001, the antiangiogenic therapies might be employed to "normalize" tumor vasculature. This approach proposes to modulate pro- and anti-angiogenic factors in order to obtain the correct balance between them and, as a consequence, the normalization of vessel structures and organization. This might result in ameliorated tumor tissue oxygenation and improved chemotherapy delivery to tumor sites [23].

In this review, we will present an updated analysis of the state of the art of anti-angiogenic therapies for glioblastoma and of the future perspectives of such therapies.

\section{History of antiangiogenic therapy for glioblastoma}

Advances in the molecular understanding of gliomagenesis have led to targeted therapies with one of the main targets being VEGF. The first inhibitor of angiogenesis approved by the Food and Drug Administration (FDA) was bevacizumab/ Avastin, a humanized monoclonal antibody targeting VEGFA isoform. It was initially approved, in 2004, for use in metastatic colorectal cancer [24]. Later on, it was approved for the treatment of non-small cell lung cancer (2006) [25], metastatic renal cell cancer (2009) [26] and glioblastoma (2009) [27].

Bevacizumab administration to glioblastoma patients results in the stabilization of tumor vasculature and in the re- 
duction of both microvascular proliferation rate and in the permeability of the blood-brain barrier (BBB) [28]. Acceptable toxicity and an immediate and dramatic reduction in the tumor enhancement on MRI scans [29] were assessed in pivotal uncontrolled phase II trials [30, 31] and were decisive factors for accelerated FDA approval of bevacizumab. Unfortunately, the initial enthusiasm, which was substantially based on an increased progression-free survival (PFS) and on the radiographic response, was frustrated by the absence of a significant improvement in overall survival (OS). This kind of pseudo- response likely reflects anti-VEGF mediated stabilization of the BBB. Two placebo-controlled phases II trials in newly-diagnosed glioblastomas soon demonstrated that bevacizumab, in addition to standard therapy, results in increased PFS without significantly enhancing OS [32, 33]. Moreover, these two studies got opposite results when considering patients' quality of life. A third study, a randomized phase II trial ("BELOB”), demonstrated increased OS in recurrent glioblastoma using the combination of bevacizumab and lomustine [34]. Following this encouraging result, a new phase III unblinded study was developed (EORTC 26101), comparing lomustine with lomustine/bevacizumab treatment on a cohort of glioblastoma patients at first relapse. The study failed to demonstrate an OS benefit in the double treatment (median OS, 9.1 months) compared with lomustine alone (8.5 months), despite an improvement in PFS from 1.5 to 4.2 months [35].

The great heterogeneity of response of glioblastoma patients to bevacizumab strongly encouraged to search for new criteria of patients stratification. Different studies have been performed to identify subclasses of patients where bevacizumab therapy could have more chances to be effective. For example, glioblastoma classification according to their subtype in The Cancer Genome Atlas led to a good correlation between tumor subtype and response to bevacizumab in terms of OS [36]. Moreover, gene expression profiling (DASL and RNA-seq) of formalin-fixed, paraffin-embedded tumor material from patients with recurrent glioblastoma participating in the BELOB trial, allowed to demonstrate that patients with classical glioblastoma subtype [37] have greater benefits from bevacizumab plus lomustine combined treatment than other glioblastoma subtypes [38]. Overall, despite the lack of a unique criterion to predict bevacizumab response and the variability of the glioblastoma patient's response to bevacizumab, a general improvement of patients' quality of life following bevacizumab therapy exists and has led FDA to admit bevacizumab full approval for recurrent glioblastoma in 2017. Conversely, European Medicine Agency (EMA) denied bevacizumab approval for recurrent glioblastoma, due to inadequate response criteria and difficulty in interpreting OS and PFS outcomes (Doc. Ref. No: EMA/70577/2010).

\section{Molecular characterization of bevacizumab-driven infil- trative shift}

Glioblastoma patients can be divided into two different groups, according to their response to bevacizumab therapy. One group exhibit intrinsic resistance to anti-angiogenic therapy and fail to show any response. Conversely, the second group is characterized by an initial phase of the response, followed by a sort of tumor adaptation, during which tumor in some way evolves and bypass angiogenic blockade [39]. There are two main hypotheses to explain the strategies the tumor adapts to circumvent the angiogenic blockade. In the pro-angiogenic pattern, the tumor activates alternative pro-angiogenic factors to support neo angiogenesis. These tumors have an increased contrast enhancement on MRI. In the pro-invasive pattern, tumors show increasing FLAIR-bright volumes indicative of more infiltrating growth. The infiltrative shift observed in the pro-invasive pattern is characterized by a peculiar phenotypic change of the tumor, which can assume a gliomatosis-like growth pattern [40, 41, 42]. At the morphological level, the infiltrative shift is characterized by vessel co-option, a phenomenon whereby the tumor cells migrate along the perivascular spaces to form multilayered sleeves. The bevacizumab-induced infiltrative shift of human glioblastoma occurs mostly along with the perivascular spaces. Moreover, using orthotopic xenograft models, it was observed an endothelium-oriented migration, mosaic tubule formation, and niching with endothelial cells, a repertoire of vascular-like behaviors by tumor cells difficult to specifically define as pro-survival response to hypoxia [43]. Bevacizumab exposure restores the BBB function of U87MG brain xenografts and human glioblastoma [44]. Coherently with this observation, bevacizumab-induced brain invasion along perivascular spaces requires the restoration of the endothelial component of BBB, which would work as a scaffold for migration [43]. Molecularly, bevacizumab triggers an epithelial to mesenchymal transition with over-expression of the receptor Plexin Domain Containing 1 (PLXDC1), in the brain xenografts of U87MG and of patient-derived glioma stem-like cells [43].

Bevacizumab-triggered metabolic alterations of glioblastoma cells were reported as well. In particular, bevacizumab increases the expression of hypoxic (HIF-1 $\alpha$ and CAIX) and glycolytic markers (GLUT1 and MCT1), leading to higher glucose uptake and lactate production [45].

Bevacizumab, which inhibits VEGF binding to VEGFRs, does not prevent binding of VEGF to the neuropilin 1 and 
2 (NPs), for long considered ancillary co-receptors of VEGFRs. However recent research, reviewed in [46], demonstrated that NPs can activate YAP/TAZ in response to VEGF independently of VEGFRs via a signaling pathway that engages integrins. In many cancers including skin, breast cancers, and medulloblastoma, VEGF-dependent, VEGFRs-independent activation of YAP/TAZ pathway promotes self-renewal of cancer stem cells and enhance cancer aggressiveness [47]. Although not explicitly demonstrated to occur in glioblastoma, such NPs signaling may explain the marginal therapeutic efficacy of bevacizumab and the bevacizumab-driven infiltrative shift. By preventing VEGF binding to VEGFRs, bevacizumab may locally increase the concentration of VEGF capable of interacting with NPs. Moreover, by binding two VEGF molecules, via the two Fab domains, bevacizumab may produce a divalent ligand for NPs thus increasing the affinity of VEGF to NPs and favoring the subsequent clustering of integrins in signaling complexes. Such a potentiating effect of antibodies against the growth factor was previously described $[48,49]$.

\section{Suppression of VEGF signaling}

VEGF subtraction is not the only way to target signaling by VEGF. A different strategy is to target VEGFRs and inhibit the receptors' tyrosine kinase activity by small molecules. Although disruption of VEGF signaling through ablation of the tyrosine activity of its receptors elicits tumor adaptation and a pro- infiltrative shift [42], as also reported for bevacizumab, a variety of molecules have been developed directed against VEGFRs. These small drugs, characterized by a range of specificity, target a variety of different tyrosine kinases (i.e. KIT, PDGFR, RET, RAF, and EGFR), although with variable affinity. Some of these small molecules have been tested in phase I/II clinical trials. Cediranib [50] is the only one of these inhibitors to be tested up to a phase III trial. Unfortunately, despite promising results from preclinical models, it did not lead to increased PFS in the Phase III REGAL (Recent in Glioblastoma Alone and With Lomustine) trial for recurrent glioblastoma, when compared to the treatment with lomustine [51]. Sunitinib and sorafenib, two tyrosine kinase inhibitors FDA-approved for metastatic renal cellcarcinoma [52], underwent phase II trials in glioblastoma, with disappointing results. Sunitinib displayed very poor anti-angiogenic activity and was highly toxic [53], while sorafenib, both alone [54] or as a combination therapy with standard care agent temozolomide, did not significantly prolong PFS [55], being at the same time highly toxic. Similarly, Pazopanib was evaluated in a single-arm phase II study in the setting of recurrent glioblastoma, showing modest responsiveness [56]. Recently, interesting indications came from Axitinib. This is an indazole derivative with an MW of 386.47, characterized by high VEGFRs specificity, blocking VEGFR-1, -2 and -3 at picomolar concentrations [57]. The IC50 is 10-fold lower for the VEGF family of receptors than for other tyrosine kinase inhibitors such as pazopanib, sunitinib, or sorafenib. However, with lower affinity, Axitinib inhibits additional kinases [58]. In vitro, Axitinib selectively blocks VEGF-stimulated receptor auto-phosphorylation leading to inhibition of endothelial cell proliferation, survival, migration and tube formation [59]. The molecule is FDA- and EMA-approved for recurrent metastatic renal cell carcinoma. Due to its strong anti-angiogenic properties, axitinib is a good candidate for anti-angiogenic therapy of glioblastoma. It indeed exhibited an anti-angiogenic and survival prolongation effect in preclinical orthotopic glioblastoma models [60]. Axitinib demonstrated anti-tumor activity with reduced toxicity in a small cohort of recurrent glioblastoma patients treated in a non-comparative randomized phase II clinical trial (AxiG-trial) [61]. Moreover, a recent second phase of the AxiG-trial provided clinical evidence that axitinib, as a monotherapy, has anti-tumor activity in patients with recurrent glioblastoma, within acceptable toxicity. There was no evidence that combination therapy of axitinib and lomustine improves the tumor response rate or survival, while the risk for hematological toxicity was increased [62].

An intriguing peculiarity of axitinib treatment in vitro is the pro-senescence effect it exerts on both tumor $[63,64]$ and endothelial cells [65]. Mechanistically, this requires oxidative stress-dependent activation of the Ataxia Telangiectasia Mutated (ATM) kinase. Axitinib-mediated senescence is prevented by short- term treatment with antioxidants (N-Acetylcysteine, NAC, or reduced glutathione, GSH), or by chemical ATM inhibitors in endothelial cells. Of note, axitinib induction of senescence seems to follow different mechanisms in transformed tumor cells and in endothelial cells, since neither antioxidants nor ATM inhibitors can prevent axitinib-induced senescent phenotype in glioblastoma tumor cells [65]. This cell-specific response could likely depend on the deregulation of ROS homeostasis in transformed cells. Irrespectively from the actual mechanism, read from a therapeutic perspective, this data might acquire a translational significance. Although specific in vivo studies are still missing, the co-administration of antioxidants together with axitinib could have clinical relevance: antioxidants might selectively protect endothelial cells from axitinib by decreasing systemic toxicity and maintaining a functional vascularization necessary for efficient delivery of chemotherapeutic drugs within the tumor mass. 


\section{Conclusions}

Glioblastoma remains one of the deadliest malignancies affecting the central nervous system, despite considerable research efforts to improve therapeutic options. Developing new strategies for the management of patients with glioblastoma is mandatory. Antiangiogenic drugs have substantially disappointed their expectations. Bevacizumab, the FDA-approved monoclonal antibody targeting VEGF, demonstrated to be effective in prolonging PFS, but substantially failed to have significant impact on OS.

Overall, forced ablation of the hypoxia response and/or of the VEGF/VEGFR pathways may trigger a phenotypic change in glioblastoma that acquires a gliomatosis-like growth pattern, characterized by more pronounced tumor invasiveness. Since only a fraction of tumors relapsing following an antiangiogenic therapy undergo this infiltrative shift, it will be crucial to find new markers for prediction of such a response, allowing accurate stratification of patients to treat with angiogenesis- targeting drugs.

\section{Conflict of interest}

The authors declare that they have no conflict of interest.

\section{Author contributions}

MPM, RP, AL, and MLF conceived the study. MLF wrote the manuscript. MPM, RP, and AL critically revised the manuscript.

\section{Acknowledgement}

This work was funded by AIRC grant IG 2019 Id.23154 to R.P.

\section{References}

1. Stupp R, Mason WP, van den Ben MJt, Weller M, et al. (2005) Radiotherapy plus concomitant and adjuvant Temozolomide for glioblastoma. N Engl J Med 352: 987-996.

2. Stupp R, Hegi ME, Mason WP, van den Bent MJ, Taphoorn MJ, et al. (2009) Effects of radiotherapy with concomitant and adjuvant temozolomide versus radiotherapy alone on survival in glioblastoma in a randomized phase III study: 5-year analysis of the EORTC-NCIC trial. Lancet Oncol 10: 459-466.

3. Louis, PerryA, Reifenberger G, vonDeimling A, Figarella-Branger D, et al. (2016) The 2016 World Health Organization Classification of Tumors of the Central Nervous System: a summary. Acta Neuropathol 131: 803-820.

4. Ohgaki H, Kleihues P (2013)The definition of primary and secondary glioblastoma Clin Cancer Res 19: 764-772.

5. Rong Y, Durden DL, VanMeir EG, Brat DJ (2006) 'Pseudopalisading' necrosis in glioblastoma: a familiar morphologic feature that links vascular pathology, hypoxia, and angiogenesis. J Neuropathol Exp Neurol 65: 529-539.

6. Folkman J (1971)Tumor angiogenesis: therapeutic implications. N Engl J Med 285: 1182-1186.

7. Baeriswyl V, Christofori G (2009) The angiogenic switch in carcinogenesis. Semin CancerBiol 19: 329-337.

8. Kerbel RS (2008) Tumor Angiogenesis. N Engl J Med 358: 2039-2049.

9. Schmidt NO, Westphal M, Hagel C, Ergün S, Stavrou D, et al. (1999) Levels of vascular endothelial growth factor, hepatocyte growth factor/scatter factor and basic fibroblast growth factor in human gliomas and their relation to angiogenesis. Int J Cancer 84:10-18.

10. Reiss Y, Machein MR, Plate KH (2005) The role of angiopoietins during angiogenesis in gliomas. Brain Pathol. 15: 311317.

11. Brat DJ, Bellail AC, Van Meir EG (2005) The role of interleukin- 8 and its receptors in gliomagenesis and tumoral angiogenesis. Neuro Oncol 7: 122-133.

12. Shibuya M (2011) Vascular Endothelial Growth Factor (VEGF) and Its Receptor (VEGFR) Signaling in Angiogenesis: A Crucial Target for Anti- and Pro-Angiogenic Therapies. Genes Cancer 22: 1097-1105.

13. Shibuya M, Claesson-Welsh L (2006) Signal transduction by VEGF receptors in the regulation of angiogenesis and lymphangiogenesis. Exp Cell Res 312: 549-560.

14. Lee S, Chen TT, Barber CL, Jordan MC, Murdock J, et al. (2007) Autocrine VEGF signaling is required for vascular homeostasis. Cell 130: 691-703. 
15. Ferrara N1, Gerber HP, Le Couter J (2003) The biology of VEGF and its receptors. Nature Med 9: 669-676.

16. Ferrara N, Kerbel RS (2005) Angiogenesis as a therapeutic target. Nature 438: 967-974.

17. Plate KH, Mennel HD (1995) Vascular morphology and angiogenesis in glial tumors. Exp Toxicol Pathol 147: 89-94.

18. Pacioni S, D'Alessandris QG, Buccarelli M, Boe A, Martini M, et al. (2019) Brain Invasion along Perivascular Spaces by Glioma Cells: Relationship with Blood-Brain Barrier. Cancers (Basel) 19:12 pii: E18.

19. Wang GL, Jiang BH, Rue EA, and Semenza GL (1995) Hypoxia-inducible factor 1 is a basic- helix-loop-helix-PAS heterodimer regulated by cellular O2 tension. Proc Natl Acad Sci USA 92: 5510-5514.

20. Xu C1, Wu X, Zhu J (2013) VEGF promotes the proliferation of human glioblastoma multiforme stem-like cells through VEGF receptor 2. Scientific World Journal 417413.

21. Bao S, Wu Q, Sathornsumetee S, Hao Y, Li Z, et al. (2006) Stem cell-like glioma cells promote tumor angiogenesis through vascular endothelial growth factor. Cancer Res 66: 7843-7848.

22. Treps L, Perret R, Edmond S, Ricard D and Gavarda J (2017) Glioblastoma stem-like cells secrete the pro-angiogenic VEGF-A factor in extracellular vesicles. J Extra cell Vesicles 6: 1359479.

23. Jain RK (2010) Normalizing tumor vasculature with anti-angiogenic therapy: a new paradigm for combination therapy. Nat Med 7: 987-989.

24. Hurwitz H, Fehrenbacher L, Novotny W, Cartwright T, Hainsworth J, et al. (2004) Bevacizumab plus irinotecan, fluorouracil, and leucovorin for metastatic colorectal cancer. N. Engl. J. Med 350: 2335-2342.

25. Cohen MH, Gootenberg J, Keegan P, Pazdur R (2007) FDA drug approval summary: bevacizumab (Avastin) plus Carboplatin and Paclitaxel as first-line treatment of advanced/metastatic recurrent nonsquamous non-small cell lung cancer. Oncologist 12: 713-718.

26. Summers J, Cohen MH, Keegan P, Pazdur R (2010) FDA drug approval summary :bevacizumab plus interferon for advanced renal cell carcinoma. Oncologist 15: 104-111.

27. Cohen MH, Shen YL, Keegan P, Pazdur R (2009) FDA drug approval summary: bevacizumab (Avastin) as treatment of recurrent glioblastoma multiforme. Oncologist 14: 1131-1138.

28. Gerstner ER, Duda DG, di Tomaso E, Ryg PA, Loeffler JS, et al. (2009) VEGF inhibitors in the treatment of cerebral edema in patients with brain cancer.Nat Rev Clin Oncol 6: 229-236. 29. Hasselbalch B, Lassen U, Hansen S, Holmberg M, Sørensen $M$, et al. (2010) Cetuximab, bevacizumab, and irinotecan for patients with primary glioblastoma and progression after ra- diation therapy and temozolomide: a phase II trial. Neuro Oncol 12: 508-516.

30. Friedman HS, Prados MD, Wen PY, Mikkelsen T, Schiff D, et al. (2008) Bevacizumab alone and in combination with irinotecan in recurrent glioblastoma. J Clin Oncol 27: 4733-4740.

31. Kreisl TN, Kim L, Moore K, Duic P, Royce C, et al. (2009) Phase II trial of single-agent bevacizumab followed by bevacizumab plus irinotecan at tumor progression in recurrent glioblastoma. J Clin Oncol 27: 740-745.

32. Gilbert MR, Dignam JJ, Armstrong TS, Wefel JS, Blumenthal DT, et al. (2014) A randomized trial of bevacizumab for newly diagnosed glioblastoma. N Engl J Med 370: 699-708.

33. Chinot OL, Wick W, Mason W, Henriksson R, Saran F, et al. (2014) Bevacizumab plus radiotherapy temozolomide for newly diagnosed glioblastoma. N Engl J Med 370: 709-722.

34. Taal W, Oosterkamp HM, Walenkamp AME, DubbinkHJ, Beerepoot LV, et al. (2014) Single-agent bevacizumab or lomustine versus a combination of bevacizumab plus lomustine in patients with recurrent glioblastoma (BELOBtrial):a randomized controlled phase2trial. Lancet Oncol 15: 943-953.

35. Wick W, Gorlia T, Bendszus M, Taphoorn M, Sahm F, et al. (2017) Lomustine and Bevacizumab in progressive glioblastoma. N Engl J Med 377: 1954-1963.

36. Sandmann T, Bourgon R, Garcia J, Li C, Cloughesy T, et al. (2015) Patients with proneural glioblastoma may derive an overall survival benefit from the addition of bevacizumab to first-line radiotherapy and Temozolomide: retrospective analysis of the AVAglio Trial. J Clin Oncol 33: 2735-2744.

37. Verhaak RG, Hoadley KA, Purdom E, Wang V, Qi Y, et al. (2010) Integrated genomic analysis identifies clinically relevant subtypes of glioblastoma characterized by abnormalities in PDGFRA, IDH1, EGFR, and NF1. Cancer Cell 17: 98-110.

38. Erdem-Eraslan L, van den Bent MJ, Hoogstrate Y, NazKhan H, Stubbs A, et al. (2016) Identification of Patients with Recurrent Glioblastoma Who May Benefit from Combined Bevacizumab and CCNU Therapy: A Report from the BELOB Trial. Cancer Res 76: 525-534.

39. Bergers G1 Hanahan D (2008) Modes of resistance to anti-angiogenic therapy. Nat Rev Cancer 8: 592-603.

40. deGroot JF, Fuller G, Kumar AJ, Piao Y, Eterovic K , et al. (2010) Tumor invasion after treatment of glioblastoma with bevacizumab: radiographic and pathologic correlation in humans and mice. Neuro Oncol 12: 233-242.

41. Gomez-Manzano C, Holash J, Fueyo J, Xu J, Conrad CA, et al. (2008) VEGF trap induces anti-glioma effect at different stages of the disease. NeuroOncol 10: 940-945.

42. Pàez-Ribes M, Allen E, Hudock J, Takeda T, Okuyama $\mathrm{H}$, et al. (2009) Antiangiogenic therapy elicit s malignant pro- 
gression of tumors to increased local invasion and distant metastasis. Cancer Cell 15: 220-231.

43. FalchettiML, D'AlessandrisQG, Pacioni S, BuccarelliM, MorganteL, et al. (2019)Glioblastoma endothelium drives bevacizumab-induced infiltrative growth via modulation of PLXDC1. Int J Cancer 144: 1331-1344.

44. Stegmayr C, Oliveira D, Niemietz N, Willuweit A, Lohmann P, et al. (2017) Influence of Bevacizumabon BloodBrain Barrier Permeability and O-(2-18FFluoroethyl)-1-TyrosineUptake in Rat Gliomas. J Nucl Med 58: 700-705.

45. Gonçalves VM, Cardoso-Carneiro D, Valbom I, Cury F. Silva VA, et al. (2017) Metabolic alterations underlying Bevacizumab therapy in glioblastoma cells. Oncotarget 8: 103657103670 .

46. Elaimy AL, Mercurio AM (2018) Convergence of VEGF and YAP/TAZ signaling: Implications for angiogenesis and cancer biology. Sci Signal. 11(552). PII: eaau1165.

47. Zanconato F, Cordenonsi M, Piccolo S (2016) YAP/ TAZ at the Roots of Cancer. Cancer Cell 29: 783-803.

48. Kara E, Dupuy L, Bouillon C, Casteret S, and Maurel MC (2019) Modulation of gonadotropins Activity by Antibodies. Front Endocrinol (Lausanne) 10: 15.

49. Schechter Y, Hernaez L, Schlessinger J \& Cuatrecasas P (1979) Local aggregation of hormone-receptor complexes is required for activation by the epidermal growth factor. Nature 278: 835-838.

50. Brave SR, Ratcliffe K, Wilson Z, James NH, Ashton S, et al. (2011) Assessing the activity of cediranib, a VEGFR-2/3 tyrosine kinase inhibitor, against VEGFR-1 and members of the structurally related PDGFR family. Mol Cancer Ther 10: 861-873.

51. Batchelor TT, Mulholl and P,Neyns B, NaborsLB, Campone, et al. (2013) PhaseIII randomized trial comparing the efficacy of cediranib as monotherapy, and in combination with lomustine, versus lomustine alone in patients with recurrent glioblastoma. J Clin Oncol 31: 3212-3118.

52. Stein MN, Flaherty KT (2007) CCR drug updates: Sorafenib and sunitinib in renal cell carcinoma. Clin Cancer Res 13: 3765-3770.

53. Hutterer M, Nowosielski M, Haybaeck J, Embacher S, Stockhammer F, et al. (2014) A single-arm phase II Austrian/ German multicenter trial on continuous daily sunitinib in primary glioblastoma at first recurrence (SURGE 01-07). Neuro Oncol 16: 92-102.

54. Hassler MR, Ackerl M, Flechl B, Sax C, Wöhrer A, et al. (2014) Sorafenib for patients with pretreated recurrent or progressive high-grade glioma: A retrospective, single-institution study. Anticancer Drugs 25: 723-728.

55. Zustovich F, Landi L, Lombardi G, Porta C, Galli L, et al.
(2013) Sorafenib plus daily low-dose temozolomide for relapsed glioblastoma: A phase II study. Anticancer Res 33: 3487-3494.

56. Iwamoto FM, Lamborn KR, Robins HI, Mehta MP, Chang SM, et al. (2010) Phase II trial of pazopanib (GW786034), an oral multi-targeted angiogenesis inhibitor, for adults with recurrent glioblastoma (North American Brain Tumor Consortium Study 06-02). Neuro Oncol 12: 855-861.

57. Escudier B, GoreM (2011) Axitinib for the management of metastatic renal cell carcinoma. Drugs R D 11: 113126.

58. Klaeger S, Heinzlmeir S, Wilhelm M, Polzer H, Vick B, et al. (2017) The target landscape of clinical kinase drugs. Science 358: pii: eaan 4368.

59. Hu-Lowe DD, Zou HY, Grazzini ML, Hallin ME, Wickman GR, et al. (2008) Nonclinical antiangiogenesis and antitumor activities of axitinib (AG-013736), an oral, potent, and selective inhibitor of vascular endothelial growth factor receptor tyrosine kinases1,2,3. Clin Cancer Res 14: 7272-7283.

60. LuL, Saha D, Martuza RL, Rabkin SD, Wakimoto H (2015)Single agent efficacy of the VEGFR kinaseinhibitoraxitinib in preclinical models of glioblastoma. J Neurooncol 121: 91-100.

61. Duerinck J, Du Four S, Vandervorst F, D'Haene N, Le Mercier M, et al. (2016) Randomized phase II study of axitinib versus physician's best alternative choice of therapy in patients with recurrent glioblastoma. J Neurooncol. 128: 147-155.

62. Duerinck S, Du Four F, Bouttens C, Andre V, Verschaeve F, et al. (2017) Randomized phase II trial comparing axitinib with the combination of axitinib and lomustine in patients with recurrent glioblastoma. Journal of Neuro-Oncology 136: 115-125.

63. Morelli MB, Amantini C, Santoni M, Soriani A, Nabissi $\mathrm{M}$, et al. (2015) Axitinib induces DNA damage response leading to senescence, mitotic catastrophe, and increased NK cell recognition in human renal carcinoma cells. Oncotarget 6: 362456259.

64. Morelli MB, Amantini C, Nabissi M, Cardinali C, Santoni M, et al. (2017). Axitinib induces senescence-associated cell death and necrosis in glioma cell lines: The proteasome inhibitor, bortezomib, potentiates axitinib-induced cytotoxicity in a p21(Waf/Cip1) dependent manner. Oncotarget 8: 3380-3395.

65. Mongiardi MP, Radice G, Piras M, Stagni V, Pacioni S, et al (2019) Axitinib exposure triggers endothelial cells senescence through ROS accumulation and ATM activation. Oncogene 38: 5413-5424. 
Submit your manuscript to a JScholar journal and benefit from:

ๆ Convenient online submission

ब Rigorous peer review

I Immediate publication on acceptance

- Open access: articles freely available online

ब High visibility within the field

- Better discount for your subsequent articles

Submit your manuscript at http://www.jscholaronline.org/submit-manuscript.php 